\title{
Ideologiekritik und Kontingenz(erfahrung) am Beispiel Fake News: Der Beitrag des Radikalen Konstruktivismus
}

Armin Scholl

Keywords: Kontingenz, Konstruktivismus, Ideologiekritik, Fake News

\begin{abstract}
Der (radikale) Konstruktivismus scheint auf den ersten Blick kein Kandidat für ideologiekritische Forschung. Wenn man sich jedoch nicht vorschnell von der Kritik am Konstruktivismus beeindrucken lässt, kann dessen erkenntniskritisches Potenzial herausgearbeitet werden. Am Beispiel von Fake News wird deutlich, dass deren Kritik nicht epistemologisch naiv von der Dichotomie Richtigkeit vs. Falschheit von Informationen und Tatsachenbehauptungen ausgehen kann, sondern eine differenziertere Argumentation erfordert. Diese Forderung wird konstruktivistisch eingelöst und abschließend kritisch geprüft.
\end{abstract}

Scholl, Armin. 2019. „Ideologiekritik und Kontingenz(erfahrung) am Beispiel Fake News: Der Beitrag des Radikalen Konstruktivismus". In Ideologie, Kritik, Öffentlichkeit. Verhandlungen des Netzwerks Kritische Kommunikationswissenschaft, herausgegeben von Uwe Krüger und Sebastian Sevignani, 46-64. Universität Leipzig. DOI: 10.36730/ideologiekritik.2019.3

Ich danke Uwe Krüger und Sebastian Sevignani für die kritischen, hilfreichen und wertvollen Hinweise.

Armin Scholl | Universität Münster | scholl@uni-muenster.de 


\section{Einleitung}

Wenn es um Fake News geht, wird schnell geurteilt, dass es richtige News gibt und absichtlich erzeugte falsche News, dass es also eine klare Trennlinie zwischen Wahrheit und propagandistischer Falschheit (nicht zu verwechseln mit unabsichtlichem Irrtum) gibt. Als der Konstruktivismus genau diese Trennlinie bezweifelt hat, indem er jede Beobachtung und mithin jede Aussage über eine Beobachtung strikt an die beobachtende Instanz gebunden hat (Schmidt 1994), hat er sich verschiedene Vorwürfe eingehandelt: Wer auf die Möglichkeit einer objektiven (im Sinn von beobachterübergreifenden oder beobachterunabhängigen) Wahrheit verzichte, mache Wissenschaft, insbesondere empirische Wissenschaft, unmöglich, da diese nur funktioniere, wenn man Theorien unabhängig an der Realität prüfen könne (vgl. Kepplinger 1990; aus materialistischer Sicht Städtler 2016, 13). Weiterhin mache er sich des Relativismus schuldig, denn wenn jegliche Beobachtung beobachterabhängig sei, sei auch jede Beobachtung prinzipiell ernst zu nehmen und habe eine gewisse Berechtigung (Hanisch 2009, 71ff.). Schließlich werde der Konstruktivismus auf diese Weise zum unfreiwilligen Helfershelfer von abstrusen Weltsichten, die sich darauf berufen könnten, dass sie eben die Wirklichkeit nach ihren Maßstäben konstruierten, seien diese auch noch so absurd in den Augen wissenschaftlich geschulter Beobachter (Sokal und Bricmont 2001, 11, 17). Insgesamt könne mit Konstruktivismus folglich alles gerechtfertigt und nichts mehr kritisiert werden.

Wenn diese Kritik am Konstruktivismus berechtigt wäre, könnte man das Vorhaben, Fake News konstruktivistisch zu verorten, bereits an dieser Stelle beenden. Ich will aber zweierlei zeigen: Zum einen ist die Behauptung einer klaren Trennlinie zwischen richtigen Fakten (und analog richtigem Bewusstsein) und falschen Fakten (und analog falschem Bewusstsein) alles andere als trivial, sondern begründungsbedürftig. Der Konstruktivismus kann recht gut darstellen, welche kognitiven Mechanismen hinter der Vorstellung von objektiv richtigen Fakten stehen. Zum anderen handelt sich der ontologische Realismus, der hinter der Philosophie des Objektivismus steht, gravierende erkenntnistheoretische und ideologische Probleme ein. Ob diese verschwinden, wenn man dialektisch vorgeht und mit einem kritischen Realismus arbeitet (vgl. Fuchs 2008), müsste extra erörtert werden. Es lohnt also ein sehr genaues Hinschauen, wie es die Phänomenologie lehrt. 
Nach einer Vorstellung der wichtigsten Charakteristika der konstruktivistischen Argumentation will ich darstellen, wie man aus konstruktivistischer Perspektive Fake News behandelt bzw. die Trennung zwischen richtigen und falschen Fakten versteht. ${ }^{1}$

Auch wenn sich eine konstruktivistische Vorgehensweise implizit gegen objektivistische, realistische, materialistische Erkenntnistheorie richtet, soll hier die Kritik am Historischen Materialismus oder an anderen objektivistischen Theorien nicht ausgeführt werden. Stattdessen will ich nachweisen, dass auch der Konstruktivismus zur Kritik an Wirklichkeitskonstruktionen in der Lage ist. Damit soll nicht nur den eingangs genannten Kritikpunkten am Konstruktivismus der Wind aus den Segeln genommen werden, vielmehr will ich darauf hinaus, dass der Konstruktivismus gar nicht unkritisch - und zwar auch im Sinn der Gesellschaftskritik, nicht nur der epistemologischen Kritik - sein darf. Oder umgekehrt: Wer mit dem Konstruktivismus Propaganda legitimiert, missbraucht den Konstruktivismus und stellt sich gegen dessen zentrale Prämissen. (In Klammern bemerkt: Vor Missbrauch ist keine Philosophie gefeit, das weiß man vom Historischen Materialismus sehr gut.)

Bevor ich jedoch die konstruktivistische Perspektive auf Ideologie und Ideologiekritik vorstelle, will ich noch kurz darauf eingehen, wo die Verbindung zur Kommunikationswissenschaft besteht, wo kommunikationswissenschaftliche Anwendungen möglich sind. Diese bestehen darin, dass die Massenmedien die Hauptproduzenten von Ideologie sind. Adorno (2002 [1944]) sprach deshalb von Kulturindustrie, um den gewerblich und fabrikartig produzierenden Charakter zum Ausdruck zu bringen. Kommunikationswissenschaftler*innen wie Horst Holzer (1973) oder Wolf D. Hund und Bärbel Kirchhoff-Hund (1980) und später Edward S. Herman und Noam Chomsky (2002) machten dies konkreter an den gesellschaftlichen Verhältnissen fest. Sie kritisierten, dass Medien als Privateigentum organisiert sind oder im Fall der öffentlich-rechtlichen Anstalten unter der staatlichen Kontrolle stehen, welche wiederum das Privateigentum schützt. Hinter dieser Vorstellung steckt der Basis-Überbau-

1 Dies ist nicht der erste Versuch, Fake News aus konstruktivistischer Perspektive zu analysieren (vgl. Herczeg 2019; Mitterer 2019; Schmidt, M. 2017; Schmidt, S.-J. 2017; Zimmermann und Kohring 2018), aber bisher wurde noch kein Anschluss an eine kritische Kommunikationswissenschaft gesucht. 
Relativismus, welcher davon ausgeht, dass die Besitz- und Produktionsverhältnisse (Basis) den ideologischen Überbau bestimmen. Relativismus nenne ich das Verhältnis deshalb, weil sich je nach historischer Verfasstheit der Besitz- und Produktionsverhältnisse (bzw. relativ dazu) die Ideologie verändert. Fällt der Klassengegensatz in einer nachrevolutionären Gesellschaft weg, müsste folglich auch die Notwendigkeit für deren ideologischen Überbau wegfallen.

Die offene Frage bleibt jedoch, ob es ideologiefreie Massenkommunikation überhaupt geben kann (derzeit kann es sie bei den herrschenden Verhältnissen nicht geben). Daraus ergeben sich Folgefragen:

- Ist ein ideologiefreier Standpunkt nötig, um einen ideologischen Standpunkt kritisieren zu können bzw. welche Prämissen sind notwendig, um Ideologiekritik betreiben zu können?

- Ist ein richtiges Bewusstsein erforderlich, um ein falsches Bewusstsein identifizieren und kritisieren zu können?

- Wie ist dieses richtige Bewusstsein herzustellen?

- Ist es objektiv, fallibel und somit kritisierbar?

- Ist dieses richtige Bewusstsein durch den (richtigen) Klassenstandpunkt zu erreichen (Stichwort Standpunktwissenschaft)?

- Kann das richtige gesellschaftliche Bewusstsein institutionalisiert werden in Form eines Systems der Massenkommunikation, oder ist eine Institutionalisierung in einer klassenlosen Gesellschaft weder notwendig noch praktizierbar?

Diese und weitere mögliche Fragen im Anschluss müssten eigentlich von einer historisch-materialistischen Theorie positiv beantwortet werden können. Wenn positive Antworten jedoch aus einer historisch-materialistischer Perspektive nicht möglich sein sollten, oder wenn man aus einer anderen Perspektive diesbezüglich keine positiven Antworten geben will, dann muss geklärt werden, ob auch bei Verneinung dieser Fragen Ideologiekritik prinzipiell möglich ist. Dies soll im Folgenden aus einer völlig anderen - nämlich konstruktivistischen - Perspektive gezeigt werden. Dabei muss offen und einer vergleichenden Analyse vorbehalten bleiben, ob eine solche reduzierte Ideologiekritik dann $\mathrm{zu}$ ähnlichen oder zu unterschiedlichen Ergebnissen führen würde. 


\section{Kontingenz(erfahrung): Die konstruktivistische Perspektive}

Im Unterschied zum erkenntnistheoretischen Realismus lässt sich der Konstruktivismus nicht auf ein emphatisches Wahrheitsverständnis ein, schon gar nicht, wenn Wahrheit als Abbild von Realität - im Unterschied etwa zum Wahrheitsverständnis als Konsens über die Wirklichkeit - aufgefasst wird. Was genau wird abgelehnt? Zum einen wird die Vorstellung von der Wahrheit von Tatsachenbehauptung als Übereinstimmung zwischen Tatsachenbehauptung und Tatsachen (Wirklichkeit bzw. Wirklichkeitsausschnitte) verworfen, weil dieser Zusammenhang zwar einen direkten Zugang zur Wirklichkeit suggeriert, aber verschleiert, dass dieser wiederum sprachlich vermittelt ist und letztlich auf der sprachlichen Reduktion (Konstruktion) von Sinneseindrücken beruht. Diese Sinneseindrücke sind ihrerseits direkt abhängig von den psychischen und biologischen Operationen des Gehirns, das diese überhaupt erst zu einem Gesamtbild zusammenfasst. Letztlich kann man aus konstruktivistischer Perspektive nur von einem unendlichen Regress sprechen; das Original die Wirklichkeit selbst - wird immer nur sinnlich-psychisch oder sprachlich-kommunikativ erfahren, sodass die Erfahrungswirklichkeit und nicht eine als ontisch gegeben angenommene Wirklichkeit die eigentlich maßgebliche Instanz für Erkenntnis ist. Ontologisch kann der Konstruktivismus folglich nur neutral bleiben, also auch nicht behaupten, es gäbe die Wirklichkeit nicht oder sie könne nicht erfahren werden, weil man das Jenseits der Erfahrung nicht überprüfen kann; es bleibt eine metaphysische Inferenz - oder kritisch ausgedrückt: eine Illusion (anschaulich bei von Glasersfeld 1987, 411-413).

Konstruktivist*innen benutzen den nicht-ontologischen, pragmatisch abgeschwächten Realitäts- und Wahrheitsbegriff der "Viabilität", um zu praktisch "gangbaren", akzeptablen Aussagen zu kommen. Dieser erkenntnistheoretische Rückzug macht es jedoch erforderlich, die Relation zwischen Erkennendem und Erkanntem stets mitzudenken und auszuweisen. Die strikte Beobachterrelativität ist das zentrale Charakteristikum des Konstruktivismus (vgl. ausführlich bei Schmidt 1987).

Im Prinzip sollte diese Position mit wissenschaftlichen Ansätzen, die sich als Standpunktwissenschaften verstehen, vereinbar sein. So nimmt Karl Marx den (Klassen-)Standpunkt des Proletariats ein, aus dessen Per- 
spektive heraus Geschichte und die Überwindung des Klassenantagonismus konzeptionell gedacht wird und in der politischen Praxis umgesetzt werden soll. Ähnlich nimmt der Feminismus die Perspektive der Frau ein, um aus dieser Position heraus die bestehenden herrschaftlichen (patriarchalischen) Geschlechterverhältnisse zu kritisieren und diese letztlich zugunsten eines emanzipatorischen Geschlechterverhältnisses zu überwinden. Dies ist bei aller Unterschiedlichkeit zwischen materialistischen Geschlechtertheorien und der sozialkonstruktivistischen Genderforschung ein gemeinsamer Nenner.

Das Problem, das sich meines Erachtens aus der strikten Standpunktbezogenheit ergibt, ist, dass dieser Standpunkt seinerseits kontingent ist. Ein universeller Wahrheitsanspruch lässt sich damit nur schwerlich begründen, will man nicht in einen Klassenimperialismus (analog zum Kulturimperialismus der westlichen Welt) verfallen. Man kann daraus folgern, dass das epistemisch „wahre“ und normativ "richtige“ (universell gemeinte und universell gültige) Bewusstsein nicht nötig ist, weil das Bewusstsein sowieso standpunktabhängig ist, biologisch, psychisch, sozial (gesellschaftlich).

Die üblicherweise hier einsetzende Kritik am (vermeintlichen) Subjektivismus, Voluntarismus und Arbitrarismus des Konstruktivismus ${ }^{2}$ ist meines Erachtens völlig überzogen. Und dies aus zwei Gründen: Zum einen ist die Erkenntnistheorie des Konstruktivismus, also deren Standpunkt, ein kollektiver Standpunkt. Dieser ist also nicht willkürlich, sondern ausgehandelt, weil er auf Vereinbarung beruht, also im besten Sinn deliberativ ist. Zum anderen ist die Reflexion des Standpunktes, also die Einnahme einer Metaperspektive statt einer blinden Ausführung des Standpunktes geradezu notwendig. Wer einen Standpunkt einnimmt und dies weiß bzw. ein Bewusstsein von dieser Standpunkthaftigkeit hat, wird von sich aus bereits zur Reflexion des Standpunktes genötigt. Abgesehen davon wird der Standpunkt aus anderen Standpunkten heraus stets kritisiert oder in Frage gestellt, wodurch ebenfalls ein Reflexions- und Legitimationszwang entsteht.

2 Die folgenden Kritikpunkte am (radikalen) Konstruktivismus werden systematisch ausgeführt in Nüse et al. (1995) und in Hanisch (2009). Die Position des (radikalen) Konstruktivismus wird in Loosen und Scholl (2017) und in Pörksen und Scholl (2011) entwickelt. 
Ein zweiter Kritikpunkt ist ebenfalls völlig überzogen, nämlich der des Relativismus: Die Einnahme eines Standpunktes impliziere, dass auch die Einnahme anderer Standpunkte genauso gut oder legitim sei, ansonsten dürfe man sich nicht auf die Standpunkthaftigkeit des eigenen Standpunktes berufen, sondern müsse universelle Geltungsansprüche erheben, welche über den eigenen Standpunkt hinausgehen. Der Denkfehler dieser Kritik besteht darin, dass Relativität mit Gleichgültigkeit (Achtung: im doppelten Wortsinn als Resignation oder als Gleichwertigkeit) gleichgesetzt wird. Aus der Unterschiedlichkeit von Standpunkten folgt jedoch keineswegs, dass alle Standpunkte die gleiche Berechtigung haben. Auch wenn es aus konstruktivistischer Perspektive keine übergeordnete, standpunktübergreifende Position gibt, da auch universalistische Geltungsansprüche jeweils an den Beobachter geknüpft sind (Glasersfeld 2009), ist gerade der Streit über die Positionen, Perspektiven und Standpunkte das, was eine vollständige Gleichheit unwahrscheinlich macht. Das bedeutet im Umkehrschluss nicht, dass sich der bessere, legitimere, menschenfreundlichere Standpunkt durchsetzt. Aber es gibt eine Chance, dass bestimmte Standpunkte überzeugender sind als andere. Man muss also nicht kalten Sozialdarwinismus unterstellen, in dem sich der Stärkere durchsetzt, denn der sich durchsetzende Standpunkt kann auch im Sinn Habermas' das bessere Argument gehabt haben.

\section{Fake News als Ideologie}

Die übliche Kritik am Konstruktivismus lautet in Bezug auf Fake News, dass diese aus konstruktivistischer Perspektive nicht als Fake News erkennbar und kritisierbar seien, weil man im Konstruktivismus nicht zwischen richtigen, wahren Tatsachen und Fake News unterscheiden könne. Dazu ist zuerst eine Begriffsklärung notwendig (vgl. Tabelle 1), die mindestens zwei Dimensionen von falschen Informationen umfasst: die Intentionalität und die Ideologie ihrer Verbreitung. Das Gegenteil von bestätigten, wahren Tatsachen ist demnach nicht (allein) Fake News, weil 
falsche Tatsachenaussagen auch einfach Fehler oder Irrtümer sein können, also unabsichtlich falsch sind. ${ }^{3}$ Unter Fake News wird üblich nur die Gruppe der Falschaussagen eingeordnet, die absichtlich herbeigeführt werden, also Falschaussagen wider besseren Wissen. Sie sind intentional falsch und nicht versehentlich falsch. Das heißt aber immer noch nicht, dass sie ausschließlich dem Zweck dienen, Propaganda für die eigene Ideologie zu machen und über die eigentlichen Kommunikationszwecke zu täuschen. Fake News werden nämlich auch satirisch zu emanzipatorischen Zwecken eingesetzt, nämlich zur Entlarvung von ideologischer Herrschaftskommunikation (Kommunikationsguerilla): Dabei werden Fakes benutzt, um Verwirrung zu stiften und herrschaftliche Kommunikationsweisen bewusst zu machen, sodass diese kritisiert und gegebenenfalls überwunden werden können.

\section{Tab. 1: Klassifizierung von falschen Informationen}

\begin{tabular}{|c|r|r|r|}
\hline \multicolumn{2}{|c|}{ falsche Informationen $\rightarrow$} & intentional & nicht-intentional \\
\hline \multirow{2}{*}{$\hookrightarrow$} & ideologisch & Propaganda (1) & Fehlverständnis, Mitläufertum (2) \\
\cline { 2 - 4 } & nicht-ideologisch & Satire/ Aufklärung (3) & Irrtum, Fehler, Versehen (4) \\
\hline
\end{tabular}

Die gegenwärtige Debatte um Fake News dreht sich stets um die Bedeutung wie in Feld 14 , wohingegen Feld 2 weitgehend unbestimmt bleibt:

3 Wie umstritten, also kontingent, die Demarkation von Irrtum und Propaganda sein kann, zeigt die merkwürdige Auffassung von Marc Zuckerberg, geäußert im Kontext der Kontroverse um das Löschen von Facebook-Beiträgen. Demnach wüssten Holocaustleugner nicht immer, dass sie im Irrtum seien, verfolgten deshalb also nicht immer propagandistische Interessen. Es ist zwar kaum nachvollziehbar, wie ausgerechnet in diesem Themenfeld Intentionalität und Propagandaabsicht vermeidbar sein soll. Zudem kann man Zuckerberg wiederum Geschäftsinteresse unterstellen, sodass das Argument ebenfalls strategisch motiviert ist. Jedoch macht das Argument deutlich, dass die Trennlinie zwischen Irrtum und Propaganda begründungsbedürftig ist und einer sorgfältigen Entwicklung von Kriterien bedarf. Quellen: https:// www.recode.net/2018/7/18/17575156/mark-zuckerberg-interview-facebook-recode-kara-swisher und http://www.spiegel.de/netzwelt/web/facebookmark-zuckerberg-will-beitraege-von-holocaust-leugnern-nicht-entfernen-a1219146.html.

4 Aus einer Synopse von Definitionen der einschlägigen Forschungsliteratur entwickeln Zimmermann und Kohring (2018) eine umfassende und kommunikationstheoretisch begründete Definition von Fake News als aktuelle Desinformation. Zu deren Kritik aus konstruktivistischer Perspektive vgl. Scholl und Völker (2019). 
Man kann unabsichtlich Propaganda weiterverbreiten, wenn die Zustimmung zu ihr einem Irrtum im Verständnis und in der Interpretation unterliegt. Nur Feld 4 ist im engeren Sinn keine Fake News, weil die Fehlerhaftigkeit der Information versehentlich und irrtümlich erfolgt.

Kompliziert wird die Debatte, wenn Fake News nicht nur in ihrer Intentionalität und in ihrer Ideologie bestimmt werden müssen, sondern wenn sie metakommunikativ als Vorwurf verwendet wird. Ausgerechnet diejenigen, die die Wahrheit, Objektivität, Transparenz, Überprüfung ihrer Tatsachenbehauptungen oder Interpretationen selbst nicht für relevant und legitimationsbedürftig halten, mithin nicht ernsthaft argumentieren (wie der US-Präsident Trump oder wie Politiker der AfD), sondern bloß propagieren, sind schnell bei der Hand, anderen Kommunikatoren das Verbreiten von Fake News vorzuwerfen („Lügenpresse“, „Lückenpresse“, „Systempresse“ ...). Der Vorwurf, Fake News zu verbreiten, wird allerdings nicht nur von Rechtspopulisten und Verschwörungsideologen benutzt, sondern auch von Regierungen und deren Ausführungsorganen (z. B. von der Polizei gegenüber Protestbewegungen), also von herrschenden Instanzen, um dissidente Positionen zu diskreditieren und zu delegitimieren. Schließlich sind auch die etablierten Medien Urheber von Fake News (etwa die Fotofälschung der BILD-Zeitung und deren Rechtfertigungsversuche, vgl. Kruse 2014).

Spätestens an dieser Stelle wird deutlich, dass der Vorwurf, Fake News zu verbreiten, selbst der Legitimation und Überprüfung mit Hilfe von empirischen Belegen im Rahmen eines praktisch-politischen Diskurses, bedarf, soll er nicht auf bloßer Autoritätsgläubigkeit (institutionalisiertes konsensualisiertes Alltagswissen) oder auf der propagandistischen Absicht der Ablenkung von eigenen argumentativen Schwächen beruhen.

Aber selbst dieser Sachverhalt ist metakommunikativ ein Politikum, weil bereits das Sich-Einlassen auf jeglichen Fake-News-Vorwurf zu einer Legitimationsspirale der Konsensaufkündigung führt. Es stellt sich zum Beispiel die Frage, ob allein die Existenz von Holocaustleugnern es notwendig macht, einen Faktencheck zum Holocaust zu erstellen und zu kommunizieren. So geschehen im schwedischen öffentlich-rechtlichen Rundfunk SVP. Dies führte nämlich zu einer politischen Metadebatte, dass gerade durch den (neuerlichen) Beweis des Selbstverständlichen und längst Bewiesenen einer Legitimationsherausforderung Entgegenkommen kommuniziert werde, was indirekt und ungewollt genau diese Position zumindest teilweise ihrerseits legitimiere: Wenn man ernsthaft die 
Fakten zum Holocaust prüft, signalisiert man, dass man den Zweiflern an diesen bereits hinreichend überprüften Fakten, also den Holocaustleugnern, zumindest insoweit entgegenkommt, als man ihren Zweifel für diskussionswürdig hält, und zwar unabhängig von der eindeutigen propagandistischen Intention. Man tut also so, als gälte es einen Fehler oder Irrtum aufzuklären, und ignoriert dabei, dass dieser vermeintliche Irrtum ausschließlich dazu dient, eine bestimmte Propaganda zu verbreiten, um sich Herrschaft zu sichern (Wolff 2018, 12). ${ }^{5}$ Es wäre zu einfach, würde man den SVP nur für seine Naivität der kostenlosen Legitimationsbeschaffung kritisieren, denn man kann pro SVP argumentieren, dass man die Holocaustleugner selbst zwar nicht mehr argumentativ erreichen kann, aber die eigentliche Zielgruppe des Faktenchecks vielmehr diejenigen sind, die von der Position der Holocaustleugner fasziniert sind oder in irgendeiner Weise damit in Berührung gekommen sind, die aber noch prinzipiell für andere Argumente und Fakten offen sind. Möglicherweise ist die Korrektur oder Nichtkorrektur von propagandistisch verbreiteten Fake News generell und nicht nur in diesem konkreten Fall ${ }^{6}$ eine Dilemmasituation, die der reflexiven Spirale der Metakommunikation geschuldet ist. Solche Metakommunikationen müssen also nicht unbedingt propagandistische Ablenkungsmanöver vom eigentlichen Sachverhalt sein, sondern können gerade diesen durch den Kontext, den sie schaffen, deutlicher zur Geltung bringen, indem sie auf die jeweilige Beobachterinstanz im Diskurs zurückverweisen. Der Konstruktivismus sensibilisiert für die Implikationen dieser Beobachterabhängigkeit:

- Die Richtigkeit von Fakten steht nicht von vornherein fest, sondern ist das Ergebnis eines (gesellschaftlichen) Diskurses. Dementsprechend sind alle Tatsachenbehauptungen kontingent, also nicht eindeutig wahr oder falsch. Holocaustleugner und Revisionisten argumentieren historisch scheinbar wissenschaftlich faktenorientiert, allerdings immer einseitig zur Unterstützung ihrer Thesen. Die den eigenen Thesen widersprechenden Fakten werden entweder nicht berücksichtigt oder so uminterpretiert, dass sie zu

5 Es kann an dieser Stelle offen bleiben, ob Holocaustleugner neben ihrer Propagandaabsicht tatsächlich an die Nichtexistenz des Holocaust bzw. der Shoah glauben oder ob sie absichtlich lügen, also Falschinformationen wider besseres Wissen verbreiten.

6 Wie man mit Fake News kritisch umgeht, darüber wird intensiv debattiert; dabei werden ethische und effektive Aspekte angesprochen (vgl. Wenzel 2019) 
den Thesen passen und sie scheinbar bestätigen. Schon aufgrund dieser propagandistischen, ideologischen, sich gegenüber Kritik immunisierenden statt wissenschaftlich prüfenden Argumentationsweise kann man an der Wahrheit der propagierten Fakten und der Wissenschaftlichkeit der Argumentation zweifeln. Die Falschheit der Tatsachenbehauptungen von Holocaustleugnern selbst ergibt sich zudem kommunikativ daraus, dass die den Holocaust beschreibende und beweisende Geschichtswissenschaft die besseren Argumente hat (glaubwürdige Zeugen und Quellen, authentisches Filmmaterial, kritisch-rationales wissenschaftliches Vorgehen usw.).

- Die Richtigkeit von Fakten lässt sich auch insofern nicht eindeutig entscheiden, als Fakten stets in einen Kontext eingebunden und damit interpretationsbedürftig sind. „Harte“ Fakten als wahre Tatsachenaussagen und ihre "weiche" Interpretation als kontingente Bedeutungszuweisungen (zu diesen Fakten) unterscheiden sich in diesem Verständnis (nur) graduell. Schon wenn der einzelne Fakt in einen Kontext gestellt wird, ist er interpretativ eingebettet: Dazu gehört bereits die Relevanz oder Hervorhebung des Fakts gegenüber anderen Fakten, also dessen Selektion aus einer Vielzahl möglicher Fakten, sowie die unterschiedlichen Möglichkeiten, den Fakt zu verstehen.

Die kategorische Trennung zwischen Fakt und Interpretation ignoriert dagegen die Beobachterabhängigkeit auch der scheinbar härtesten Fakten oder hält sie für irrelevant. Der Konsens über Fakten ist nichts anderes als das Produkt des Konsenses über die Tatsache und ihre Interpretation oder anders: Als Tatsachen akzeptierte Fakten sind zum Konsens gewordene Interpretationen. Kontingenz kann diesen Konsens jederzeit in Frage stellen. Die Legitimation dieser Kontingenz ist wiederum im praktischen Diskurs zu klären (siehe das obige Beispiel zum Faktencheck in Bezug auf den Holocaust). Der interpretatorische Toleranzbereich oder Spielraum ist beim Rekurs auf Fakten sicher enger als bei Meinungskundgaben ${ }^{7}$, jedoch sind auch Fakten durch Perspektivität 
gekennzeichnet, die kommunikativ im Diskurs thematisiert und problematisiert werden kann.

- Beobachtung meint im konstruktivistischen Verständnis zweierlei: zum einen Distanz zum Beobachteten und den Beobachtern, zum anderen Involviertheit in den Zusammenhang von Beobachtung und Beobachtungsergebnis. Demnach ist auch die Beobachtung des Beobachters beim Beobachten, also die Beobachtung zweiter Ordnung, eine Beobachtung, also abhängig vom Standpunkt des Beobachters und damit keinesfalls eine privilegierte, höherwertige Form des Beobachtens. ${ }^{8}$ Durch die Reflexivität des Beobachtens tritt aber die Relationalität des Beobachtens zumindest punktuell in Erscheinung. In Bezug auf die Beurteilung von Fake News impliziert dies, dass man nicht vorschnell Fakten für wahr halten kann, die mehrheitlich so gesehen werden oder die der eigenen Einstellung bzw. Überzeugung entsprechen. Der Konstruktivismus macht deutlich, dass das Für-Wahr-Halten von Fakten von zahlreichen Faktoren abhängt, die dieses als Produkt der Wahrheitsattribution bestimmen. Methodisch führt diese Position weg vom Vergleich zwischen Tatsachenbehauptung und Tatsache selbst (Korrespondenz) hin zur Beobachtung von Tatsachenbehauptung (Kohärenz, Konsens).

Dies bedeutet aber nicht, dass Konsens oder Kohärenz als hinreichende Ersatzkriterien für Korrespondenz als Wahrheitskriterien gelten können, denn man kann sich auch kollektiv irren oder kohärent dogmatisch argumentieren. Das konstruktivistische Ersatzkriterium für Wahrheit, Viabilität, bleibt also notwendig vage und latent, weil die Perspektive und der interpretative Spielraum auch von Fakten einbezogen werden. Damit ist aber gerade nicht eine willkürliche Vagheit und beliebige Konstruktion impliziert, sonst

dass auch Deutungen bzw. Interpretationen mehr oder weniger angemessen sein können.

8 Gerade diese zweite Eigenschaft von Beobachtung als Verstrickung und Involviertheit wird in der Kritik am Konstruktivismus gerne ignoriert. Die Ausführungen von Maturana und Varela $(1992,255)$ hierzu sind aber eindeutig, dass der Beobachter stets ein verstrickter Beobachter ist. Daraus hat sich ein eigener Zweig des Konstruktivismus entwickelt, der als „enactivism“ bezeichnet wird (vgl. Varela et al. 1992). 
wäre die Faktizität im Diskurs nicht gegenüber Fiktionalität abgrenzbar. Viabilität meint damit nur, dass im Diskurs vorläufig (zeitlich) von vielen Diskursteilnehmer*innen (sozial) bestimmte Fakten (sachlich) als richtig akzeptiert werden. Es ist also eher ein empirisches Kriterium zur Beschreibung eines Diskurses als ein normatives Kriterium zur Regulierung des Diskurses, wenngleich es auch normativen Druck entfalten kann, weil ein permanentes Bezweifeln von Fakten im Diskurs eher als strategisches Manöver angesehen wird (wie es typisch ist für das Verhalten von Trollen).

- Bei faktischen Behauptungen wird im Diskurs in der Regel auf die Beobachterreferenz verzichtet, weil und wenn davon ausgegangen wird, dass Fakten (im Unterschied zu Meinungen) unabhängig von spezifischen und partikularen Beobachterpositionen wahr sind, sodass der Verweis auf die Beobachterposition redundant oder sogar schädlich für die Behauptung selbst wäre. Warum müsste man sonst auf die Beobachterabhängigkeit hinweisen, wenn man nicht von der Allgemeingültigkeit der Fakten überzeugt wäre? Hier käme also eine falsche Subjektivierung eines an sich objektiven Sachverhalts ins Spiel. Aus konstruktivistischer Perspektive kann man sich durchaus darauf einlassen, den Beobachterstandpunkt bei Fakten nicht permanent mitzukommunizieren („Ich behaupte, dass X." statt "X ist ...").

Darin besteht der pragmatische (im Sinn von praktische und wirkungsbezogene) Charakter des Konstruktivismus: Der Alltagsrealismus der Beobachtung erster Ordnung wird nicht geleugnet oder abgelehnt, weil Wirklichkeitskonstruktionen oft Common Sense und unproblematisch sind. Sobald jedoch entweder die Faktizität der faktischen Aussage oder deren Wahrheitsgehalt in Frage gestellt wird, wird die Referenz sichtbar und notwendig. Man muss dann Gründe, die für die Wahrheit der Behauptung sprechen, angeben (können). Wenn in einem Diskurs über Fakten und deren Faktizität der Vorwurf der Fake News eingebracht wird, reden wir nicht mehr über die unterstellte Falschheit allein, sondern zusätzlich über den Ideologieverdacht der absichtlichen Fälschung, Manipulation, Täuschung und Propagandaabsicht dessen, der Fake News produziert und verbreitet. 
Der Konstruktivismus ist demnach eine konsequent soziale bzw. sozialwissenschaftliche Erkenntnistheorie, weil er nichts außerhalb des Individuellen und Sozialen benötigt, um Erkenntnis zu begründen. Vielmehr entlarvt er Wahrheitsemphase durch die Position des Wahrheitspragmatismus ${ }^{9}$. Dies ist deshalb aber kein richtiges Bewusstsein. Und umgekehrt ist falsches Bewusstsein, also Ideologie, immer beobachterabhängig, wenngleich nicht jede beobachterabhängige Beobachtung als Ideologie kritisiert werden muss und darf. Der Konstruktivismus kann und will folglich nicht selbst darüber urteilen, welches Gedankengut als ideologisch charakterisiert werden kann. Er erlaubt allerdings die Vermutung und den Verdacht von Ideologie, wenn bestimmte argumentative Bedingungen vorliegen, die man als strikte Leugnung der Kontingenz (TINA: There is no alternative) und der Begründungspflicht des eigenen Standpunkts zusammenfassen kann. Dies ist typisch für Propaganda. Das Urteil über den Ideologiegehalt einer Argumentation ist dann dem praktisch-politischen Diskurs vorbehalten.

Das Kritische des Konstruktivismus besteht darin, dass er Beobachtungen als solche (empirisch) registriert, was nicht bedeutet, (normativ) legitimiert. Es wird also nicht von vornherein eine bestimmte Beobachterperspektive selektiert, priorisiert, favorisiert und damit selbst der Kritik entzogen. Umgekehrt ist diese Kritik auch nicht bereits dadurch legitim, dass sie geäußert wird. Insofern kann auch die Kritik der Kritik unterzogen werden, was man als Reflexivierung von Kritik bezeichnen könnte. Der Konstruktivismus gibt keine normativen Maßstäbe vor, wonach diese Kritik vorzugehen habe, wie man Recht im Diskurs bekommen könne usw. Er sensibilisiert nur für die Fallstricke der Beobachterabhängigkeit und der Kontingenz von Beobachtungen. Der Umgang mit Kontingenz bzw. mit Kontingenzerfahrung kann für ein erweitertes Verständnis von Kritik ebenfalls ein Beitrag zur Aufklärung und Emanzipation sein.

9 Der Begriff des Pragmatismus sollte nicht mit „Leichtfertigkeit“ oder mit einer Haltung des „Es-nicht-so-genau-Nehmens“ verwechselt werden, sondern in seinem philosophischen Kontext als praktische Wirksamkeit verstanden werden. 


\section{Konstruktivistische Lehren und Probleme}

Das pragmatische Viabilitätskriterium im Radikalen Konstruktivismus schwächt das emphatische Wahrheitskriterium der Korrespondenztheorie in zweierlei Hinsicht ab: Zum einen benötigt es nicht die metaphysische Voraussetzung der direkten Zugänglichkeit einer ontisch vorgegebenen Wirklichkeit; zum anderen lässt es verschiedene, mitunter sogar widersprüchliche Wahrheitswerte zu, sofern diese pragmatisch zum Ziel führen. Man muss Viabilität deshalb aber nicht utilitaristisch deuten, da es sich um ein Diskursprodukt handelt, das kommunikativ zustande kommt und so seine Wirkung entfaltet. Es ist widerspruchssensibel und nicht durchsetzungsorientiert.

Wenn registriert wird, dass es selbst zu hochgradig konsensfähigen Tatsachenbehauptungen noch Widersprüche gibt, heißt das nicht, diese auch zu legitimieren, sondern es ist ein Aufruf zur Prüfung und Legitimierung aller Beobachterstandpunkte, inklusive der begründeten Verweigerung von Legitimierung wie am Beispiel des schwedischen Rundfunks gezeigt werden sollte.

Die Auseinandersetzung mit Fake News als einem Gegenstand im praktischen Diskurs sollte deutlich machen, dass Konstruktivismus durch den konsequenten Einbezug der Beobachtungsinstanz die Reflexion der Beobachtung und deren Kommunikation erzwingt. Tatsachenbehauptungen werden dadurch unter Legitimationsdruck gebracht und bedürfen der Beweisführung (die sich allerdings nicht auf die bloße Behauptung von Evidenzen beschränken kann und darf). Durch den so entstehenden kommunikativen Aufwand wird Zeit „gewonnen“ (für die Reflexion) oder „verbraucht" (auf Kosten der praktischen Handlung). Daraus entsteht zugleich die Stärke des Konstruktivismus, dass er den rationalen Diskurs befördert, wie eine Schwäche, insofern als dieser Diskurs zum Selbstzweck ausarten kann - diese Ambivalenz teilt der Konstruktivismus mit Habermas ${ }^{`}$ Diskurstheorie. Es stellt sich nämlich ganz praktisch die Frage, inwiefern überhaupt ein Diskurs über bestimmte Positionen geführt werden soll, wenn oder weil diese Positionen unmittelbar als propagandistisch erkennbar sind und in einem Diskurs zur Klärung von Wahrheitsansprüchen theoretisch wie praktisch deplatziert sind. Selbstverständlich sollte ein Diskurs nicht mutwillig oder voreilig abgebrochen werden. Wenn der Verdacht aufkommt, dass es nur um Propaganda statt um Ar- 
gumentation geht, sollte vielmehr zunächst und zumindest versucht werden, dies in einem Meta-Diskurs (Diskurs über Diskursregeln, Diskursziele usw.) zu klären.

Der Konstruktivismus ist folglich insofern hilfreich, als er durch die erkenntnistheoretische Fundierung immer wieder für die Konstruktion von Gewohnheiten, Naivitäten, Dogmen usw. sensibilisiert und sie damit kontingent macht. Er liefert jedoch keine externen Kriterien für den Streit um erkenntnistheoretische Fragen, sondern verweist auf die Verantwortlichkeit der Beobachtungsinstanz für ihre Beobachtungen zurück, ist also eine bloße Selbstsensibilisierungstheorie und keine Handlungsentlastungsinstitution. Letztlich hängt es vom praktischen Gebrauch des Konstruktivismus ab, ob man sich in praktischen Diskursen durch die Verwicklung in Pseudoargumentationen instrumentalisieren lässt und damit ungewollt und indirekt affirmativ handelt, was man jedoch erst einmal erkennen muss. Oder ob man den Konstruktivismus als Kritikinstrument benutzt und das Ideologische an einer Argumentation herausarbeitet und damit emanzipatorisch handelt.

Hieran lassen sich beispielhaft herrschaftskritische Anschlussfragen und -probleme theoretisch und empirisch behandeln:

- Wenn Fake News ein generelles gesellschaftliches Klima der epistemischen Unsicherheit erzeugen, welche Machtmechanismen stecken dahinter?

- Wenn durch Fake News eine Spirale der epistemischen Konsensaufkündigung in Gang gesetzt wird, wie lässt sich ein Diskurs etablieren, der die Strategie propagandistischer Herrschaftsausübung zumindest einhegen kann oder ein Gegengewicht zu ihr erzeugen kann?

- Welche epistemische und politische Rolle spielen Einrichtungen, die Faktenchecks betreiben?

Eine kritisch-theoretisch angeleitete kommunikationswissenschaftliche Analyse solcher Problemfelder könnte konstruktivistisch inspiriert und bereichert werden, ohne ihren kritischen Gehalt einzubüßen. Ernst von Glasersfeld (2009) hat seinen (radikalen) Konstruktivismus nicht ohne Grund in die Nähe des erkenntnistheoretischen (und implizit auch des politischen) Anarchismus gerückt. 


\section{Literatur}

Fuchs, Christian. 2008. „Sociology, Dynamic Critical Realism, and Radical Constructivism. Open Peer Commentary on Ernst von Glasersfeld: Who Conceives of Society?" Constructivist Foundations 3 (2): 97-99. https:// constructivist.info/3/2/059.glasersfeld. Zugegriffen: 15. November 2019.

Glasersfeld, Ernst von. 1987. „Siegener Gespräche über Radikalen Konstruktivismus“. In Der Diskurs des Radikalen Konstruktivismus, herausgegeben von Siegfried J. Schmidt, 401440. Frankfurt am Main: Suhrkamp.

Glasersfeld, Ernst von. 2009. „Relativism, Fascism, and the Question of Ethics in Constructivism". Constructivist Foundations 4 (3): 117-120.

Hanisch, Detlef Arthur. 2009. Darstellung und Kritik des Konstruktivismus aus kritisch-rationaler Perspektive. Zur Frage nach der Existenz der Realität und ihrer objektiven Erkennbarkeit. Frankfurt am Main: Peter Lang.

Herczeg, Petra. 2019. „Wie wirklich sind die Fakten? Von der De-konstruktion zur Fälschung". In Radikaler Konstruktivismus. Vergangenheit, Gegenwart und Zukunft. Ernst von Glasersfeld (1917-2010), herausgegeben von Theo Hug, Josef Mitterer und Michael Schorner, 231-248. Innsbruck: Innsbruck University Press.

Herman, Edward S., und Noam Chomsky. 2002. Manufacturing Consent. The Political Economy of the Mass Media. New York: Pantheon Books.

Holzer, Horst. 1973. Kommunikationssoziologie. Reinbek bei Hamburg: Rowohlt.

Horkheimer, Max, und Theodor W. Adorno. 2002 [1944]. Dialektik der Aufklärung. Philosophische Fragmente (Erstauflage 1944). Frankfurt am Main: Fischer.

Hund, Wulf D., und Bärbel Kirchhoff-Hund. 1980. Soziologie der Kommunikation. Reinbek bei Hamburg: Rowohlt.

Kepplinger, Hans Mathias. 1990. „Realität, Realitätsdarstellung und Medienwirkung“. In Fortschritte der Publizistikwissenschaft, herausgegeben von Jürgen Wilke, 39-55. Freiburg, München: Verlag Karl Alber.

Krüger, Uwe. 2018. „Fake News und Propaganda als Themen für die Kommunikationswissenschaft". Aviso 66, 7-8. https://www.dgpuk.de/sites/default/files/Aviso\%20_66_01-2018_0.pdf. Zugegriffen: 15. November 2019.

Kruse, Tobias. 2014. Ein Schlagstock macht Schlagzeilen. Trugbilder, Teil II: Ein bisschen beschneiden, ein paar suggestive Begriffe - und schon hat ein Foto eine neue Wirkung. Die Bild-Zeitung zeigt, wie es geht. Fluter vom 21.11.2014, https:/ / www.fluter.de/einschlagstock-macht-schlagzeilen. Zugegriffen: 15. November 2019.

Loosen, Wiebke, und Armin Scholl. 2017. „Journalismus und (algorithmische) Wirklichkeitskonstruktion. Epistemologische Beobachtungen". Medien \& Kommunikationswissenschaft 65 (2): 348-366.

Maturana, Humberto R., und Francisco J. Varela. 1992. The Tree of Knowledge: The Biological Roots of Human Understanding (revised edition). Boston (Mass): Shambhala Publications. 
Mitterer, Josef. 2019. „The Fake News Debate. Brief Comments“. In Radikaler Konstruktivismus. Vergangenheit, Gegenwart und Zukunft. Ernst von Glasersfeld (1917-2010), herausgegeben von Theo Hug, Josef Mitterer \& Michael Schorner, 217-230. Innsbruck: Innsbruck University Press.

Nüse, Ralf, Norbert Groeben, Burkhard Freitag, und Margrit Schreier. 1995. Über die Erfindungen des radikalen Konstruktivismus. Kritische Gegenargumente aus psychologischer Sicht. Weinheim: Deutscher Studien-Verlag.

Pörksen, Bernhard, und Armin Scholl. 2011. „Entgrenzung des Journalismus: Analysen eines Mikro-Meso-Makro-Problems aus der Perspektive der konstruktivistischen Systemtheorie“. In Ebenen der Kommunikation. Mikro-Meso-Makro-Links in der Kommunikationswissenschaft, herausgegeben von Thorsten Quandt und Bertram Scheufele, 25-53. Wiesbaden: VS Verlag für Sozialwissenschaften.

Schmidt, Malte G. 2017. „Back to the Future. (Popmusik-)Journalismus im neuen faktischen Zeitalter (Journalism on pop music in the new factual era)“. medien $\mathcal{E}$ zeit: Kommunikation in Vergangenheit und Gegenwart 32 (4): 34-47.

Schmidt, Siegfried J. 1987. „Der Radikale Konstruktivismus: Ein neues Paradigma im interdisziplinären Diskurs“. In Der Diskurs des Radikalen Konstruktivismus, herausgegeben von Siegfried J. Schmidt, 11-88. Frankfurt am Main: Suhrkamp.

Schmidt, Siegfried J. 1994. „Die Wirklichkeit des Beobachters“. In Die Wirklichkeit der Medien. Eine Einführung in die Kommunikationswissenschaft, herausgegeben von Klaus Merten, Siegfried J. Schmidt und Siegfried Weischenberg, 3-19. Opladen: Westdeutscher Verlag.

Schmidt, Siegfried J. 2017. „Konstruktivismus: ein Pate des Fake_Ismus?“ medien E zeit: Kommunikation in Vergangenheit und Gegenwart 32 (4): 33.

Scholl, Armin, und Julia Völker. 2019. „Fake News, aktuelle Desinformation und das Problem der Systematisierung. Anmerkungen zum Aufsatz von Fabian Zimmermann \& Matthias Kohring ,'Fake News' als aktuelle Desinformation - systematische Bestimmung eines heterogenen Begriffs'." Medien \& Kommunikationswissenschaft 67 (2): 206214.

Sokal, Alan, und Jean Bricmont. 2001. Eleganter Unsinn. Wie die Denker der Postmoderne die Wissenschaften mißbrauchen. München: dtv.

Städtler, Michael. 2016. „Materialistische Grundlagen kritischer Gesellschaftstheorie“. In Aufsätze zur Ideologiekritik, herausgegeben vom AStA der Uni Münster, 13-27. Münster: AStA-Druckerei.

Varela, Francisco J., Evan Thompson, und Eleanor Rosch. 1992. The Embodied Mind: Cognitive Science and Human Experience. Cambridge (Mass), London: MIT Press.

Wenzel, Andrea. 2019. „To Verify or to Disengage: Coping with 'Fake News' and Ambiguity”. International Journal of Communication 13, 1977-1995.

Wolff, Reinhard. 2018. Gibt es zum Holocaust noch was zu klären? taz vom 16. Juli 2018, 12.

Zimmermann, Fabian, und Matthias Kohring. 2018. „'Fake News' als aktuelle Desinformation. Systematische Bestimmung eines heterogenen Begriffs". Medien E Kommunikationswissenschaft 68 (4): 526-541. 


\section{Open Access}

Dieser Beitrag erscheint unter der Creative-Commons-Lizenz CC-BY-SA 4.0: https://creativecommons.org/licenses/by-sa/4.0/ 